\title{
Light scalar mesons and the data on two-kaon correlation functions
}

\author{
Nikolay N. Achasov ${ }^{1, *}$ and Alexey V. Kiselev ${ }^{1,2, * *}$ \\ ${ }^{1}$ Sobolev Institute for Mathematics, Novosibirsk, 630090 \\ ${ }^{2}$ Novosibirsk State University, Novosibirsk, 630090
}

\begin{abstract}
It is shown that the ALICE data on the $K_{S}^{0} K^{+}$correlation in $\mathrm{Pb}-\mathrm{Pb}$ interactions together with the data on the $\gamma \gamma \rightarrow \eta \pi^{0}$ and $\phi \rightarrow \eta \pi^{0} \gamma$ reactions could be simultaneously described when $a_{0}(980)$ has no constituent $q \bar{q}$ component at all. Besides, the validity of the Gaussian assumption is kept.
\end{abstract}

It is proposed the nonet of light scalar mesons: $a_{0}(980), f_{0}(980), \sigma(600), \kappa(800)$ [1]. $a_{0}(980)$ and $f_{0}(980)$ mesons are well-established parts of this nonet. They were discovered $\sim$ 50 years ago and became hard problem for the naive quark model from the outset. Elucidation of their nature can shed light on confinement and the chiral symmetry realization way in the low energy region, that is why $a_{0}(980)$ and $f_{0}(980)$ became one of the central problems of nonperturbative QCD. Note that perturbation theory and sum rules don't work here. Many experimental and theoretical papers have been devoted to these particles.

In 2015 it was presented [2] a simultaneous description the Belle data on $\gamma \gamma \rightarrow \eta \pi^{0}$ [3] and the KLOE data on $\phi \rightarrow \eta \pi^{0} \gamma$ [4], signal reactions are $\gamma \gamma \rightarrow a_{0}^{0}(980)+a_{0}^{\prime 0} \rightarrow \eta \pi^{0}$ and $\phi \rightarrow\left[a_{0}^{0}(980)+a_{0}^{\prime 0}\right] \gamma \rightarrow \eta \pi^{0} \gamma$, where $a_{0}^{\prime}$ is a scalar meson with mass about $1400 \mathrm{MeV}$. The reaction $\gamma^{*}\left(Q^{2}\right) \gamma \rightarrow \eta \pi^{0}$ was also considered there.

In Ref. [5] we took into account the new data on $K_{S}^{0} K^{+}$correlation in $\mathrm{Pb}-\mathrm{Pb}$ interactions [6]. Two-particle correlation $C(Q)$ could be defined as

$$
C(Q)=\frac{A(Q)}{B(Q)}
$$

Here $A(Q)$ represents the distribution of the invariant relative momentum $Q=\sqrt{-q^{\mu} q_{\mu}}$, $q^{\mu}=p_{1}^{\mu}-p_{2}^{\mu}$, for a pair of particles from the same event. $B(Q)$ is a reference distribution of the pairs of particles taken from the different events.

In the approach based on Ref. [7] the $K_{S}^{0} K^{+}$correlation $C\left(k^{*}\right)$ is

$$
C\left(k^{*}\right)=1+\frac{\lambda}{2}\left(\frac{1}{2}\left|\frac{f\left(k^{*}\right)}{R}\right|^{2}+2 \frac{\operatorname{Re} f\left(k^{*}\right)}{\sqrt{\pi} R} F_{1}\left(2 k^{*} R\right)-\frac{\operatorname{Im} f\left(k^{*}\right)}{R} F_{2}\left(2 k^{*} R\right)\right),
$$

where $k^{*}$ is the kaon momentum in the kaon pair rest frame

$$
k^{*}=\frac{\sqrt{\left(s-\left(m_{K_{S}^{0}}-m_{K^{+}}\right)^{2}\right)\left(s-\left(m_{K_{S}^{0}}+m_{K^{+}}\right)^{2}\right)}}{2 \sqrt{s}},
$$

\footnotetext{
*e-mail: achasov@math.nsc.ru

**e-mail: kiselev@math.nsc.ru
} 
$s$ is the invariant two-kaon mass squared, $R$ is the radius parameter from the spherical Gaussian source distribution, $\lambda$ is the correlation strength, and

$$
F_{1}(z)=\frac{e^{-z^{2}}}{z} \int_{0}^{z} e^{x^{2}} d x, \quad F_{2}(z)=\frac{1-e^{-z^{2}}}{z} .
$$

The scattering amplitude used by experimenters is

$$
f\left(k^{*}\right)=\frac{\gamma_{a_{0} \rightarrow K \bar{K}}}{m_{a_{0}}^{2}-s-i\left(\gamma_{a_{0} \rightarrow K \bar{K}} k^{*}+\gamma_{a_{0} \rightarrow \pi \eta} k_{\pi \eta}\right)} .
$$

Here the denominator is the inverse propagator of the $a_{0}^{ \pm}$in the Flatté-like form [8]. Let's remind that the narrow width approximation does not work in case of $a_{0}(980)$ and $f_{0}(980)$, so the Flatté propagator is not adequate for the $f_{0}(980)$ and $a_{0}(980)$ study, see, for example, Ref. [9]. We use

$$
f\left(k^{*}\right)=\frac{2}{\sqrt{s}} \sum_{S, S^{\prime}} \frac{g_{S K_{S}^{0} K^{+}} G_{S S^{\prime}}^{-1} g_{S^{\prime} K_{S}^{0} K^{+}}}{16 \pi} .
$$

where $S, S^{\prime}=a_{0}^{+}, a_{0}^{\prime+}$, and the constants $g_{S K_{S}^{0} K^{+}}=-g_{S K_{L}^{0} K^{+}}=g_{S K^{+} K^{-}}$. The matrix of the inverse propagators is

$$
G_{S S^{\prime}} \equiv G_{S S^{\prime}}(m)=\left(\begin{array}{cc}
D_{a_{0}^{\prime}}(m) & -\prod_{a_{0}^{\prime} a_{0}}(m) \\
-\prod_{a_{0}^{\prime} a_{0}}(m) & D_{a_{0}}(m)
\end{array}\right),
$$

see details in Ref. [5] and references therein.

We have found a simultaneous description of $\gamma \gamma \rightarrow \eta \pi^{0}$ and $\phi \rightarrow \eta \pi^{0} \gamma$ reactions together with $K_{S}^{0} K^{+}$correlation when $a_{0}(980)$ has no constituent $q \bar{q}$ component at all $\left(g_{a_{0} \gamma \gamma}^{(0)}=0\right.$, $\left.g_{d \bar{u} a_{0}^{-}}=g_{u \bar{u} a_{0}^{0}}=g_{d \bar{d} a_{0}^{0}}=0\right)$ and $\lambda=1$, providing the validity of the Gaussian assumption. Some properties of the resonances in this variant are shown in Table, the curves are on Figs. 1, 2. The $a_{0}(980)$ coupling constants in Table are within the framework of the fourquark model scenario. Note these results appeared in Ref. [10] and differ from the results presented in Ref. [5].

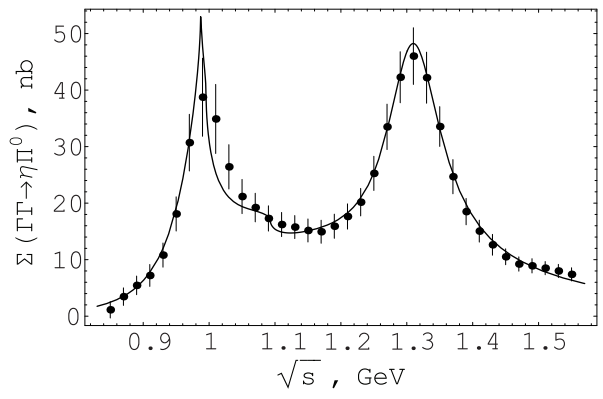

(a)

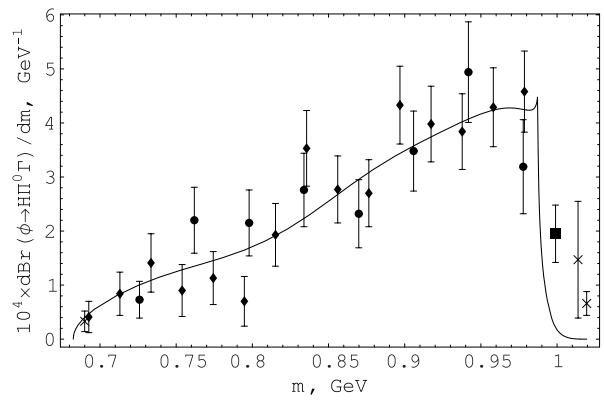

(b)

Figure 1. (a) The $\gamma \gamma \rightarrow \eta \pi^{0}$ cross section, our curve and the experimental data [3]. (b) The $\phi \rightarrow \eta \pi^{0} \gamma$ decay, cross points are omitted in the fitting. The data is from [4]. 


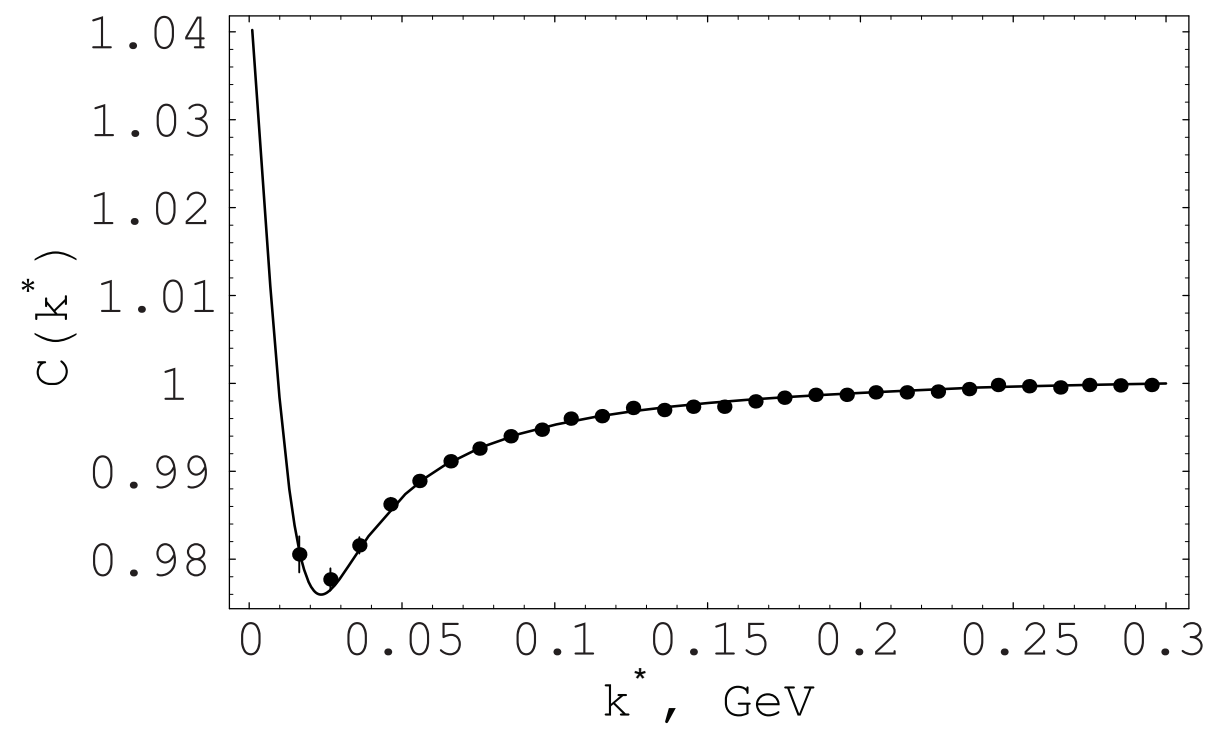

Figure 2. $K_{S}^{0} K^{+}$correlation: the solid line represents our fit, and the points are the experimental data [6].

\begin{tabular}{ll|ll}
\multicolumn{5}{c}{ Properties of the resonances and the description quality } \\
\hline$m_{a_{0}}, \mathrm{MeV}$ & 999.5 & $m_{a_{0}^{\prime}}, \mathrm{MeV}$ & 1439.4 \\
\hline$g_{a_{0}^{0} K^{+} K^{-}}, \mathrm{GeV}$ & 3.50 & $g_{a_{0}^{\prime 0} K^{+} K^{-}}, \mathrm{GeV}$ & 4.45 \\
\hline$g_{a_{0} \eta \pi}, \mathrm{GeV}$ & 3.42 & $g_{a_{0}^{\prime} \eta \pi}, \mathrm{GeV}$ & -0.20 \\
\hline$g_{a_{0} \eta^{\prime} \pi}, \mathrm{GeV}$ & -3.64 & $g_{a_{0}^{\prime} \eta^{\prime} \pi}, \mathrm{GeV}$ & 0.41 \\
\hline$g_{a_{0}^{0} \gamma \gamma}^{(0)}$ & 0 & $g_{a_{0}^{\prime 0} \gamma \gamma}^{(0)}, 10^{-3} \mathrm{GeV}^{-1}$ & -14.62 \\
\hline$\lambda$ & 1 & $R, \mathrm{fm}$ & 6.3 \\
\hline$\chi_{s p}^{2} / 24$ points & 25.3 & $\chi_{c o r r}^{2} / 29$ points & 17.2 \\
\hline$\chi_{\gamma \gamma}^{2} / 36$ points & 10.9 & $\left(\chi_{\gamma \gamma}^{2}+\chi_{s p}^{2}+\chi_{c o r r}^{2}\right) /$ n.d.f. & $55.5 / 75$ \\
\hline
\end{tabular}

Brief summary and conclusions:

1. Experimental study of two-kaon correlations is a valuable source of information about light scalar mesons.

2. New experimental data on two-kaon correlations support the four-quark model of $a_{0}(980)$.

3. The data description with $g_{a_{0}^{0} \gamma \gamma}^{(0)}=0$ is presented for the first time, meaning that $a_{0}(980)$ has no constituent $q \bar{q}$ component.

4. Fixation $\lambda=1$ in this description provides the validity of the Gaussian assumption.

The work was supported by the program of fundamental scientific researches of the SB RAS No. II.15.1., project No. 0314-2016-0021. The present work is partially supported by the Russian Foundation for Basic Research Grant No. 16-02-00065 and the Presidium of the Russian Academy of Sciences Project Grant No. 0314-2015-0011. 


\section{References}

[1] C. Patrignani et al. (Particle Data Group), Chin. Phys. C 40, 100001 (2016) and 2017 update.

[2] N.N. Achasov, A.V. Kiselev, and G.N. Shestakov, Phys. At. Nucl. 79, 397 (2016).

[3] S. Uehara et al. (Belle Collaboration), Phys. Rev. D 80, 032001 (2009).

[4] A. Aloisio et al. (KLOE Collaboration) Phys. Lett. B 536, 209 (2002).

[5] N.N. Achasov and A.V. Kiselev, Phys. Rev. D 97, 036015 (2018).

[6] S. Acharya et al. (ALICE Collaboration), Phys. Lett. B 774, 64 (2017).

[7] R. Lednicky, V. Lyuboshits, Sov. J. Nucl. Phys. 35, 770 (1982).

[8] S.M. Flatté, Phys. Lett. B 63, 224 (1976).

[9] N.N. Achasov and V.V. Gubin, JETP. Lett. 62, 191 (1995); Phys. Lett. B 363, 106 (1995).

[10] N.N. Achasov and A.V. Kiselev, arXiv:1805.10145. 